\title{
Energy Efficient Cross-layer Routing Protocols for IoT Applications - An Empirical Research
}

\author{
Aditya Tandon, Prakash Srivastava
}

\begin{abstract}
The Internet of Things (IoT) is viewed as one of the quickest cum-developing advances giving a deep rooted arrangement towards getting to the moderate and clean vitality around the globe however has dependably requested a cross-layer way to deal with handle the basic prerequisites of each layer actualized in TCP/IP convention suite. The point of this examination paper is to convey a concise audit of vitality productive cross-layer steering conventions for IoT systems. The paper enrolls novel commitments by differed analysts over the world concerning steering conventions proposed. Moreover, the paper likewise displays relative investigation of directing conventions based on specialized details principally concentrating on vitality productivity. It was likewise discovered that a large portion of these conventions did not have the highlights of security and portability since they depended on either Wireless Sensor Networks (WSNs) or Mobile Ad hoc Networks (MANETs).
\end{abstract}

Catchphrases: WSN, routing, cross-layer, Internet of Things, energy efficient.

\section{INTRODUCTION}

Internet of Things (IoT) is an equivalent word of an unending number of inserted electronic gadgets that are interconnected to build up the mankind by giving different activation, detecting and correspondence administrations. IoT gadgets are little and its ordinarily worked on batteries and different other vitality sources. With the innovation of the Wireless Sensor Networks (WSNs), the technology has been too much investigated for the last decade and alongside a horde of the steering instruments, different ways to deal with diminish the bundle and casing size of the Medium Access Control (MAC) and Physical (PHY) layers have been displayed by the specialists. Numerous such undefined components have been made vitality mindful; collection with combination techniques conveyed; timing, area and security instruments are advancing the fundamental frameworks; abnormal state deliberations bolstered with working framework plans and huge scale the board frameworks for taking care of the information that has been made in a worthy methodology. We are additionally now seeing the independence in the vitality the executives for the IoT systems.

Since a few IoT gadgets have remote associations, the requirement for higher volume remote systems is high. As of now, the remote systems take a shot at the authorized Industrial, Scientific, and Medical (ISM) groups, yet

Revised Manuscript Received on August 14, 2019. Amity University, Noida, U.P, India. (Email: askaditya@ieee.org)

Prakash Srivastava, Department of Computer Science and Engineering, Amity University, Noida, U.P, India. (Email: psrivastava9@amity.edu)
Aditya Tandon, Department of Computer Science and Engineering,

regularly don't satisfy the guidelines and as an answer, the creators in proposed the Dynamic Spectrum Access (DSA) and sharing as a savvy and high yield technique for the expanding requests. Since, IoT will utilize numerous gadgets for imparting between the objective clients; there might be heaps of transfer speed being squandered. This must be controlled, and an ideal measure of information should stream to the essential areas. For this to occur, there are a few structures and calculations that might be utilized for steering.

These conventions are known as steering conventions and they can control the information move in the correspondence ways between the nodes of the system. These conventions help in productive correspondence between the switches in this manner expanding the general comprehension of the system. It will most likely under-stand the measure of information required by every node and guarantees distribution of required information in the predefined node [46]. These conventions are otherwise called steering arrangements. Numerous way steering conventions might be utilized as it is incredibly testing to ensure the vitality utilization. Be that as it may, even this isn't altogether enough since just the bundles in the information are considered for adjusting the nodes. Subsequently, there won't be sufficiently any data to know the genuine measure of information that is transmitted between the nodes. To unravel this detriment, cross layer configuration can be utilized where another steering convention is utilized perused the correspondence between the system layer and the MAC layer. Cross layer steering conventions are kinds of conventions, where the structure does not really pursue a solitary request of execution. Rather, the system bounces between the layers at whatever point vital for better improvement of information between the nodes. This arrangement of conventions helps in making data accessible to various dimensions in the stack. This system of dynamic access and sharing is by and large provided food for vitality productive application in IoT where the transfers from differing sensor nodes proliferate the information toward a door that interfaces an overall system, for example, the Internet. Be that as it may, consolidating different structure viewpoints like the shorter correspondence run between topographically found articles, restricted vitality imperatives, and the lower preparing power into the steering convention understands the IoT model [17].

WSNs assume a critical job in the execution of IoT vision their conduct resembles an advanced skin and the sending of a virtual layer guarantees that the computational frameworks can proficiently peruse the data of the physical [13]. The 


\section{ENERGY EFFICIENT CROSS-LAYER ROUTING PROTOCOLS FOR IOT APPLICATIONS - AN EMPIRICAL RESEARCH}

terms - security and protection turned into the prime components of IoT because of unbound nature of remote correspondence. Different activities from physical layer to the application layer are improved by the convention stack intended for IoT condition. To meet the prerequisites of specific layers, various working gatherings are made for planning the conventions [44]. Different bunching and steering calculations were proposed for the development of information bundle as it needs to go enormous separations to achieve the goal and the calculations diminished time required and upgraded vitality productivity, and along these lines, it was simple for the gate crashers to follow and discover the situation of nodes amid the correspondence and release the common data [33].

Organization of the survey

The second section of the paper covers different cross layer steering conventions professional presented and actualized till date. The third section plots a portion of the potential uses of IoT including cross-layer instruments. The fourth section features a portion of the issues and difficulties by and by confronted. And the final section draws out the ends from the writing survey done as such far and the future scope of the paper.

\section{CROSS-LAYER ROUTING MECHANISMS}

Minimization of vitality has been a consuming issue for basically occasion based frameworks, for example, the WSNs, and they frequently rely upon deliberate exertion of different constantly watching miniaturized scale sensor nodes that watches a physical wonder. Heterogeneous cross layer directing conventions for WSNs in IoT applications have been characterized based on various execution measurements, for example, number of heterogeneity level, security, bundle estimate, vitality effectiveness, and so forth. Figure 1 portrays the scientific categorization of such conventions and further, these components are quickly clarified. Different cross layer steering strategies can be arranged into five gatherings - grouping based, booking based, topology based, area based, and vitality situated. In any case, these instruments were proposed over the flow decade after the examination network began to really comprehend and actualize the IoT according to the OSI or the DoD (TCP/IP) reference model. Their chronology of the advancement has been shown in Figure 2 .

In the beginning of the current decade, the authors imagined IoT as the excogitated variation of MANETs. The recreations were done on NS-2.34 [3] utilizing CBR traffic with the underlying realized Internet interfacing nodes forget about to be 5 of 20 associations [33]

In the next year, the author Young-Jun Chung proposed a vitality productive and vitality mindful directing for unicast correspondences for WSNs dependent on the AODV steering convention [34]. The model recreations were directed in the NS-2 which brought about accomplishing a moderately slight increment in the system lifetime for example the nodes' batteries were wearing out somewhat later than the AODV and MinER steering conventions.

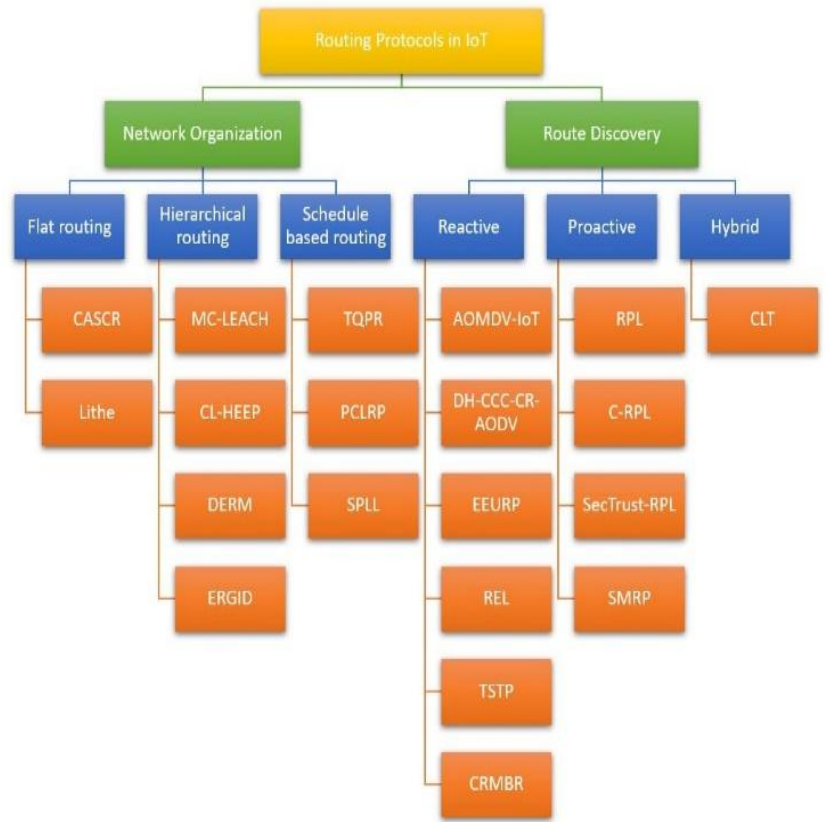

Fig. 1. Depiction of categorization of different cross layer routing protocols studied.

The researchers in [35] published their proposed work utilized three information structures put away inside the nodes viz. ECDT, CDT and SCDT where the terms E and S were mentioned as Environment and Subordinate respectively. The additional terms $-\mathrm{D}$ is information, $\mathrm{C}$ is setting, and $\mathrm{T}$ is table (see Figure 4). The MATLAB [2] reproduction results demonstrate that their proposed system accomplishes better execution in correlation with LEACH and SPT directing conventions.

Machado et al. proposed a steering convention relying upon Link and Energy quality, which was used for applications in IoT condition [14] (see Figure 5). The model recreations were managed and ran on OMNET++ test system [4] utilizing the Castalia structure and the outcomes when looked at AODV and LABILE demonstrate the administration accessibility and system lifetime.

A year prior to the official introduction of the Constrained Application Protocol (CoAP) by the Internet Engineering Task Force (IETF) that when the institutionalization was in procedure of revision, the researchers in [24] introduced Lithe, an amalgamation of CoAP and the Datagram Transport Layer Security (DTLS) in the IoT paradigm which displayed repeated IPv6 Header Compressions and Fragmentations. Legitimate additions were identified when the Contiki-Cooja [1] re-enactment results were assessed concerning the system wide reaction time at whatever point the compacted DTLS was empowered. Parameters, for example, the parcel measure, measure of vitality expended, and time taken for handling for represented the presentation assessment. 
reppesentedtheppresentatoon assesments

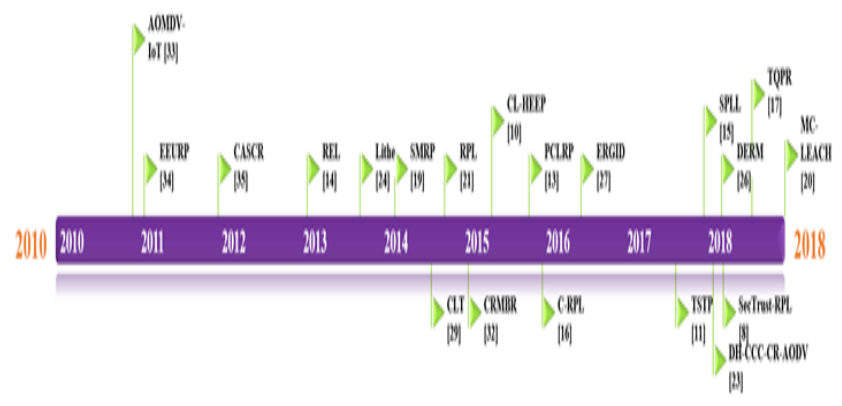

Fig. 2. Chronology of the Routing Protocols over the present decade

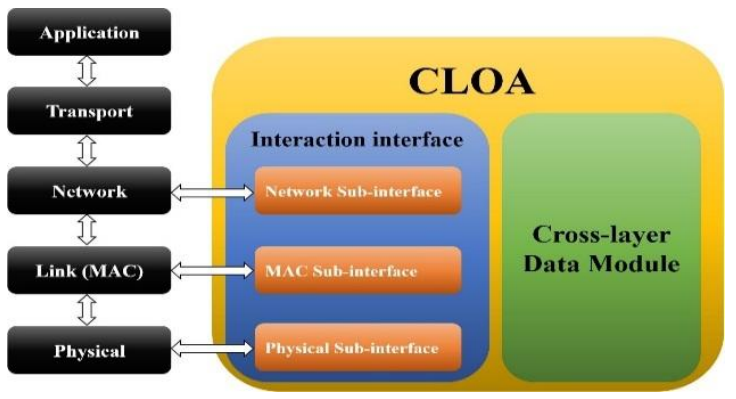

Fig. 3. CLA for the IoT Protocol Suite

Here, the idea of utilizing an encoded record (EF) amazed the authors in [19] which gave them the plan to center upon the security part of the IoT with the end goal that each system must enlist their presently running or proposed application(s), the system tends to it covers and various information connect areas to a brought together framework, which was named as Service Provider (SP).

The proposed work in [29] emphasizes on a planned trust exertion among the nodes while lessening battery corruption and overabundance memory wastage in the nodes. Building up a trust history with every single neighboring node by conveying a sliding window framework accomplishes the referenced oddity. This component was presented by the authors in [21] in which the IPv6 when packed and its header when divided opened a novel and lightweight correspondence plan for the gadgets with low power and lossy interchanges because of the correspondence and power overheads. This component utilizes the Destination Oriented Directed Acyclic Graph (DODAG) and after that concludes just as stipends "positions" to every node.

The authors in [32] proposed a steering instrument dependent on portability which worked in Cognitive Radio empowered Mobile Ad hoc Networks (MANETs). This empowered the exchange of the subjective data, for example, accessible transmission capacity from the physical-layer to the MAC and system layers and the divert quality in an intermittent way by presenting a cross-layered substructure. The recreated environments of the CRMBR were contrasted and AODV utilizing the OPNET software [5].
The authors in [10] proposed the component which empowered the alteration of transmission control, so the vitality saves in multi bounce condition of the WSN be spared. The paper utilized the system design and named it as the Cross Layer Optimization Agent (CLOA) which is shown in Figure 3.

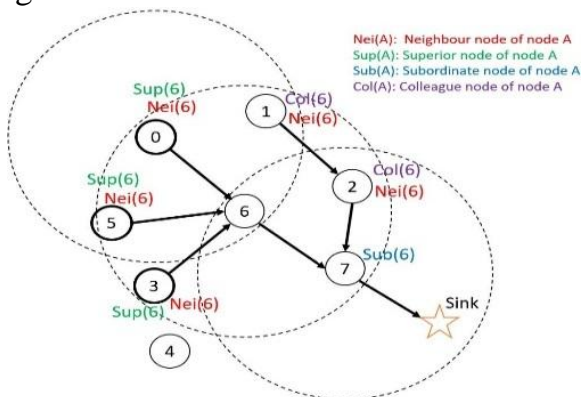

Fig. 4. Node nomenclature in an IoT/WSN network

This recently acquainted course data was utilized with change the transmission control which propelled a crosslayer obligation cycle. To spare vitality, an advantageous radio for a wake-up took up the activity. The recreations uncovered that it is vitality productive with a significant presentation upgrade when compared with different conventions.

The researchers in [13] exhibited their work on the said instrument which facilitates with the MAC layer and executes a similar need - based methodology as it does with the system layer for human services applications. PCLRP and PCLMAC joined guarantees the system traffic dispersal to be solid and the correspondence channel is tweaked for getting to the inside the-node just as "between the node" "forward and backward" messages. The re-enactment results demonstrate that the PCLRP can achieve a custom fitted QoS and altogether outflanks different systems when the presentation parameters, for example, control utilization, bundle conveyance proportion and postpone become an integral factor.

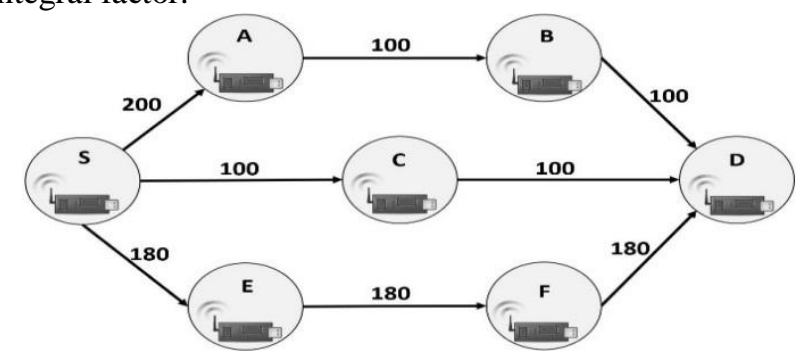

Fig. 5. Network Diagram for E2E link quality estimation in REL protocol

Barcelo et al. [16] proposed this directing convention which utilized a joined methodology for producing a few events among the nodes and made it simple to do as such. The MATLAB re-enactments uncovered that a superior exchange off between the vitality productivity and the other exhibition parameters could be practiced when contrasted 


\section{ENERGY EFFICIENT CROSS-LAYER ROUTING PROTOCOLS FOR IOT APPLICATIONS - AN EMPIRICAL RESEARCH}

with the RPL for heterogenous IoT networks.

Qiu et al. [27] proposed a proficient steering convention with the expectation of execution improvement if there should arise an occurrence of predictable information transmission and productive crisis reaction in IoT network. The recreation results demonstrate that SPEED and the EASPEED compared by ERGID in different variables like misfortune in parcels, start to finish delay (E2E) and vitality utilization.

The authors [11] introduced a cross-layer structure of the TSTP. It was planned to convey coordinated, scrambled and believed which can be effectively conveyed to a sink or passage together with the information messages (when geologically referenced). With the assistance of such game plan including various systems administration benefits in a solitary correspondence substructure, the said instrument can wipe out the information replication over every one of the administrations and in this manner an accomplishing little overhead in regard to control messages.

The said system was proposed by the Farhan et al. [15] with the expect to think of a vitality - arranged way grouping and for sensor empowering remote system condition containing message planning calculations. The proposition approach includes the compelling co-activity between way arrangement and message booking by contemplating the way connects, message sender area, and number of processors in a sensor node.

TABBEI

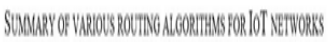

\begin{tabular}{|c|c|c|c|c|c|c|c|c|}
\hline Protorol & Simularer tyd & $\begin{array}{l}\text { linery } \\
\text { inthichy }\end{array}$ & $\begin{array}{l}\text { Combers. } \\
\text { amarenses }\end{array}$ & Seruility & $\begin{array}{l}\text { Nalli } \\
\text { lupp } \\
\text { rowling }\end{array}$ & 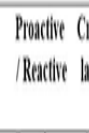 & & Iolility \\
\hline 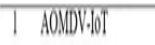 & 15:? & INditum & No & No & $Y_{18}^{\circ}$ & & & Yes \\
\hline : EERP & NSS: & Metiun & No & it & 10 & Rextive & 10 & No \\
\hline CASCR & NATAB & Whetion & $Y_{6}$ & 16 & $Y_{6}$ & Prodicie & No & $Y_{6}$ \\
\hline I REL & OWNEIH & Indiun & $Y_{6}$ & 16 & $Y_{6}$ & Prostin 1 & $Y_{B S}$ & 10 \\
\hline I lithe & Contibiccowas & INefium & No & 怡 & No & Prowiere & 10 & No \\
\hline 6 SMLPP & Nonere & Lort & 10 & $Y_{0}$ & $V_{0}$ & Prodicie 1 & Yos & $Y_{6 s}$ \\
\hline 1 (LI & NSS: & INefium & No & Y苗 & 10 & Prowirie & 10 & No \\
\hline 8 RM & OWEETH & INefium & $Y_{\text {Y }}$ & Y & Y & Prowiri 1 & Yes & $Y_{\text {期 }}$ \\
\hline ) CRBBR & ONEET & Lorr & No & 10 & Y & Prodicie & 10 & $Y_{G}$ \\
\hline 10 (LANEP & 19:? & Hilgh & $\mathrm{No}$ & No & Y & Powaine & Yis & $Y_{\text {Y }}$ \\
\hline || PCLPP & OANEEH & Netium & No & 10 & Y & Prodiari & Yis & No \\
\hline 12 CRL & MATLAB & Lorl & No & It & 10 & Prowier & Yos & Y⿻ \\
\hline I) ERGID & 15:2 & Hing & No & No & $Y_{0}$ & Rextive & YW & Y的 \\
\hline 14 ISTP & OWNET+ & Lorr & No & $Y_{*}$ & 10 & Proditive 1 & Yis & No \\
\hline IS SPLL & MATLAB & Metium & No & 16 & Yis & Protier & 10 & $Y_{G}$ \\
\hline 16 DHCCC.CR :000 & NS: & Larr & in & it & $\gamma_{0}$ & Rexation & No & $Y_{\text {S }}$ \\
\hline II DERI & 15:? & Nodium & No & in & $\gamma_{0}$ & Prasion & Yos & No \\
\hline 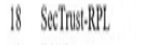 & 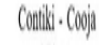 & MNevina & No & $Y_{6}$ & $Y_{B}$ & Prowiont & & $Y_{B}$ \\
\hline 19 TQPR & NSS? & Lar & No & No & No & Prodive 1 & Yes & No \\
\hline 20 NCCEACH & MATAB & Lorr & No & 10 & No & Rextivit I & Yis & $Y_{H B}$ \\
\hline
\end{tabular}

The authors in [23] proposed the Cognitive Radio (CR) AODV steering convention which they professed to work under half duplex radio handset, and they named it as Directional Hybrid-CCC-CR-AODV directing convention. This system was conveyed with the authorized access arrange where the intellectual clients worked on the Primary User (PU) range extended when the said steering instrument was presented for numerous jumps presented by the name Multi-hop Cluster LEACH (MC-LEACH) calculation. Figure 3 delineates thegroups at whatever point these PU nodes are idle with the $\mathrm{CR}$ dimensional range.

The creators likewise demanded in utilizing the recommended E2E authorized PU free channels together with the course disclosure amid RREQ/RREP messages so as to deal with the IoT obliged information at the system layer. The reproduction results examined in the paper guarantee that the exhibition of proposed convention alongside the directional control and information transmission improve the achievable throughput and decrease node and system vitality usage when contrasted and other existing CR-AODV steering conventions.

$\mathrm{Wu}$ et al. [26] proposed a postpone mindful steering calculation for WSNs with a way fixed portable sink and it is additionally vitality effective, named DERM, which strikes an alluring balance between sparing vitality and conveying dormancy. The creators clarify the proposed DERM structure and extensively assess the calculation by standing out it from two accepted directing calculations and thus, a standard arrangement is introduced. After far reaching assessment, the outcomes recommend that parcel of vitality sparing might be given by the proposed strategy while likewise maintaining a higher conveyance proportion and meeting the defer imperatives.

With the constant motivation and clear aim towards a protected IoT, the researchers in [8] proposed the framework which gives assurance against Sybil and Rank assaults by installing it into the RPL. A trust - based structure advances the exhibition of the system by identifying and disengaging the assaults. The recreations results demonstrate that its presentation is moderately worthy while contrasting and others in the comparable classification and this was indicated utilizing the testbed tries moreover.

Natarajan et al. [17] talked about the said directing plan with the goal to acquire slight however huge changes in the application layer to produce the crisis and typical information parcels, the system layer towards way grouping dependent on criticism and booking plans for a specific time quantum which was created in the MAC layer.

Yarde et al. [20] exhibited a cross-layer directing arrangement of guidelines for WSN and IoT. A specialized worldview was extended when the said steering instrument was presented for numerous jumps presented by the name Multihop Cluster LEACH (MC-LEACH) calculation. Figure 3 delineates the composition of a cross-layer operator which interfaces with the initial three layers of the IoT convention stack.

\section{PLAUSIBLE UTILIZATIONS}

There are multitude of applications that the cross-layered mechanisms empower the IoT paradigm. 


\section{A. Enhancing Security}

1) Secured IoT Gateway for Smart Applications [22]:

This paper described the cross-layered architecture comprising a Key Management System (KMS) and a Wireless Device (WD) in order to achieve effective security authentication. This specific arrangement was implemented on Raspberry-Pis as secured IoT gateways and several field tests were conducted for three IoT applications namely Manufacturing, eHealth monitoring and an Integrated Social-Sensing solution using Vehicle-to-Anything (V2X) network. The intent of this paper was to enable such deployments depending heavily on peer- to-peer communications.

2) Biometric Recognition System for Mobile IoT devices [28]:

This paper proposed a cross-layer biometric recognition system for mobile IoT devices. The authors ensure minimal computational complexity by divide-and-conquer approach. They insist on separating the developments of hard- ware and software parts of the system to achieve better security against different intrusion attempts by presenting the effect of Hardware Trojans (HTs) on the design parameters such as critical path and area duration time. The algorithm gives the confusion matrix output which is included in the software part.

\section{3) Traffic Management in Social Internet-of-} Vehicles (SIoV) [6]:

This research intended to contain the congestion problem in Vehicular Ad hoc Networks (VANETs) when SIoV was implemented to serve better traffic management in a city. This paper proposed an empirical cross-layer architecture for congestion control based on Ring structure to segregate different vehicles across different areas in the city.

\section{B. Industrial IoT Applications}

1) Scheduling solutions [31]:

This work makes use of the latest IETF IPv6 over IEEE $802.15 .4 \mathrm{e}$ TSCH mode (6TiSCH). for cost-effective industrial monitoring and control applications. To achieve stringent communication requisites of several industrial applications, mesh networks based on IEEE 802.15.4 were introduced in 2015, named as Time Synchronized Channel Hopping (TSCH). The authors imagined a scenario of IIoT implemented in a wireless multi-hop mesh network. Their CONCISE solution to the problem enabled the routing and segregation of the data in a content specific approach via a deterministic TSCH scheduling to achieve better communication reliability and reduce E2E latency.

2) Forensic Investigation in Critical Infrastructure (CI) applications [7]:

This paper describes the forensics of IoT devices while specifically focusing on the state-of-the-art challenges with the Industrial IoT subset. The authors have taken the use case of the United States criminal justice system stressing on the inadequacy in dealing with cybercrime cases, especially concerning about the IIoT device attacks in CI applications such as Industrial Control System / Supervisory Control and Data Acquisition (ICS/SCADA) applications

\section{ISSUES AND CHALLENGES \& RESULTS}

Till date there have been the exploration cerebral pains of vitality effectiveness, security and portability. A conceivable answer for various issues according to the relative examination is the cross-layer approach which improves the exhibition in IoT as it gives different capacities separated from steering, similar to the power productivity, by including both the MAC and PHY layers. So as to think about and investigate the conventions, it is important to examine them in detail. Table I represents the similar investigation of different cross layer steering conventions in IoT including com-mon parameters, for example, vitality proficiency, setting mindfulness, security, multi-bounce and whether they are proactive or responsive directing instruments. A mechanized learning process has been utilized for guaranteeing that an ideal spread of vitality use is performed. Henceforth, it is important to make an exchange off between the vitality lop-sidedness and vitality productivity.

\section{CONCLUSION AND FUTURE SCOPE}

It has been seen that every one of the proposed systems have their own benefits and negative marks and accordingly, a vitality effective cross-layered directing convention must associate with various layers (MAC and PHY). This is done so as to expand the hibernation time of the nodes which are not utilized in the steering procedure, disposing of the wastage of vitality caused because of ceaseless use of trivial nodes, limiting the utilization of a similar way to the bundles in the course, guaranteeing that the nodes don't wake up before the planned restart time and plunging the impacts between the ways

Cross-layer configuration expresses that the recovery and tweaking the parameters having at least two layers accomplishes characterized objectives. Researches on these steering conventions in IoT are as yet in progress and along these lines, a ton of research inspirations still persevere around there. This paper has a solid idea that a large portion and will likewise care for certain security concerns being looked by the IoT gadgets today. This half breed topology must be a blend of the talked about conventions. The applications might be utilized while thinking about the conventions for the cross breed calculation. While, every one of these conventions has just been executed for IoT applications, it is basic to locate the most appropriate half and half topology, which might be considered for the future work.

\section{REFERENCES}

1. "Cooja Simulator - Contiki" [Online]. Available: https://anrg.usc.edu/contiki/index.php/Cooja_Simulator.

2. "MathWorks - Makers of MATLAB and Simulink MATLAB \& Simulink" [Online]. Available: https://in.mathworks.com/. 
3. "Network Simulator ns-2" [Online]. Available: http://nsnam.sourceforge.net/wiki/index.php/User_Infor mation.

4. "OMNeT++ Discrete Event Simulator" [Online]. Available: https://omnetpp.org/.

5. "OPNET Technologies - Network Simulator | Riverbed" [Online]. Available: https://www.riverbed.com/in/products/steelcentral/opnet. html.

6. B. Jain, G. Brar, J. Malhotra, S. Rani, and S. H. Ahmed, "A cross layer protocol for traffic management in Social Internet of Vehicles," Future Generation Computer Systems, vol. 82, pp. 707-714, 2018.

7. C. M. Rondeau, M. A. Temple, and J. Lopez, "Industrial IoT cross- layer forensic investigation," Wiley Interdisciplinary Reviews: Forensic Science, vol. 1, no. 1, pp. e1322, 2019.

8. D. Airehrour, J. A. Gutierrez, and S. K. Ray, "SecTrust-RPL: A secure trust-aware RPL routing protocol for Internet of Things," Future Generation Computer Systems, vol. 93, pp. 860-876, 2019.

9. D. Cacciagrano, R. Culmone, M. Micheletti, and L. Mostarda, "Energy-Efficient Clustering for Wireless Sensor Devices in Internet of Things," Performability in Internet of Things, pp. 59-80, 2019.

10. D. E. Boubiche, A. Bilami, S. Boubiche, and F. Hidoussi, "A cross-layer communication protocol with transmission power adjustment for energy saving in multi-hop MhWSNs," Wireless Personal Communications, vol. 85, no. 1, pp. 151-177, 2015.

11. D. Resner and A. A. Fröhlich, "Design rationale of a cross-layer, trustful space-time protocol for wireless sensor networks," in 2015 IEEE 20th Conference on Emerging Technologies \& Factory Automation (ETFA), pp. 1-8, 2015.

12. F. Al-Turjman, "Cognitive routing protocol for disasterSystems, vol. 92, pp. 1103-1115, 2019.

13. H. B. Elhadj, J. Elias, L. Chaari, and L. Kamoun, "A priority based cross layer routing protocol for healthcare applications," Ad Hoc Networks, vol. 42, pp. 1-18, 2016.

14. K. Machado, D. Rosário, E. Cerqueira, A. Loureiro, A. Neto, and J. de Souza, "A routing protocol based on applications," Sensors, vol. 13, no. 2, pp. 1942-1964, 2013.

15. L. Farhan, R. Kharel, O. Kaiwartya, M. Hammoudeh, and B. Adebisi, "Towards green computing for Internet of things: Energy oriented path and message scheduling approach," Sustainable Cities and Society, vol. 38, pp. 195-204, 2018.

16. M. Barcelo, A. Correa, J. L. Vicario, and A. Morell, "Cooperative interaction among multiple RPL instances in wireless sensor networks," Computer Communications, vol. 81, pp. 61-71, 2016

17. M. Natarajan and S. Subramanian, "A cross-layer design: energy efficient multilevel dynamic feedback scheduling in wireless sensor networks using deadline aware active time quantum for environmental monitoring," International Journal of Electronics, vol. 106, no. 1, pp. 87-108, 2019.

18. P. L. R. Chze and K. S. Leong, "A secure multi-hop routing for IoT communication," in 2014 IEEE World Forum on Internet of Things (WF-IoT), 2014, pp. 428432.

19. P. L. R. Chze, K. S. Leong, A. K. Wee, E. Sim, K. E. May, Y. J. Jie, and H. S. Wing, "Secured IoT Gateway For Smart Nation Applications," in 2018 14th International Wireless Communications \& Mobile Computing Conference (IWCMC), 2018, pp. 1065-1068.

20. P. Yarde, S. Srivastava, and K. Garg, "A Cross-Layer inspired internet of things," Future Generation Computer energy and link quality for internet of things

Routing Protocol for Wireless Sensor Networks," Data and Communication Networks, pp. 83-91, 2019.

21. Q. Le, T. Ngo-Quynh, and T. Magedanz, "RPL-based multipath routing protocols for Internet of Things on wireless sensor networks," in 2014 International Conference on Advanced Technologies for Communications (ATC 2014), 2014, pp. 424-429.

22. R. Kaur, K. Verma, S. K. Jain, and N. Kesswani, "Efficient Routing Protocol for Location Privacy Preserving in Internet of Things," International Journal of Information Security and Privacy (IJISP), vol. 13, no. 1, pp. 70-85, 2019.

23. S. Anamalamudi, A. R. Sangi, M. Alkatheiri, and A. M. Ahmed, "AODV routing protocol for Cognitive radio access based Internet of Things (IoT)," Future Generation Computer Systems, vol. 83, pp. 228-238, 2018.

24. S. Raza, H. Shafagh, K. Hewage, R. Hummen, and T. Voigt, "Lithe: Lightweight secure CoAP for the internet of things," IEEE Sensors Journal, vol. 13, no. 10, pp. 3711-3720, 2013.

25. S. Taheri and J.-S. Yuan, "A Cross-Layer Biometric Recognition System for Mobile IoT Devices," Electronics, vol. 7, no. 2, pp. 26, 2018.

26. S. Wu, W. Chou, J. Niu, and M. Guizani, "Delay-aware energy-efficient routing towards a path-fixed mobile sink in industrial wireless sensor networks," Sensors, vol. 18, no. 3, pp. 899, 2018.

27. T. Qiu, Y. Lv, F. Xia, N. Chen, J. Wan, and A. Tolba, "ERGID: An efficient routing protocol for emergency response Internet of Things," Journal of Network and Computer Applications, vol. 72, pp. 104-112, 2016.

28. T. Umer, M. H. Rehmani, A. E. Kamal, and L Mihaylova, "Information and resource management systems for Internet of Things: Energy management, communication protocols and future applications,," Future Generation Computer Systems, vol. 92, pp. 1021$1027,2019$.

29. X. Anita, M. A. Bhagyaveni, and J. M. L. Manickam, "Collaborative lightweight trust management scheme for wireless sensor networks," Wireless Personal Communications, vol. 80, no. 1, pp. 117-140, 2015.

30. Y. Jahir, M. Atiquzzaman, H. Refai, A. Paranjothi, and P. G. LoPresti, "Routing protocols and architecture for Disaster Area Network: A survey," Ad Hoc Networks, vol. 82, pp. 1-14, 2019.

31. Y. Jin, U. Raza, A. Aijaz, M. Sooriyabandara, and S. Gormus, "Content centric cross-layer scheduling for industrial IoT applications using 6TiSCH," IEEE Access, vol. 6, pp. 234-244, 2017.

32. Y. Sun, J. Bai, H. Zhang, R. Sun, and C. Phillips, "A Mobility- Based Routing Protocol for CR Enabled Mobile Ad Hoc Networks," International Journal of Wireless Networks and Broadband Technologies (IJWNBT), vol. 4, no. 1, pp. 81-104, 2015.

33. Y. Tian and R. Hou, "An improved AOMDV routing protocol for internet of things," in 2010 International Conference on Computational Intelligence and Software Engineering, 2010, pp. 1-4.

34. Y. Chung, "An energy-efficient unicast routing protocol for wireless sensor Networks," Tech. Int. J. Comput. Sci. Emerg. Tech, vol. 2, pp. 60-64, 2011.

35. Z. Chen, H. Wang, Y. Liu, F. Bu, and Z. Wei, "A context-aware routing protocol on internet of things based on sea computing model, " Journal of Computers, vol. 7, no. 1, pp. 96-105, 2012.

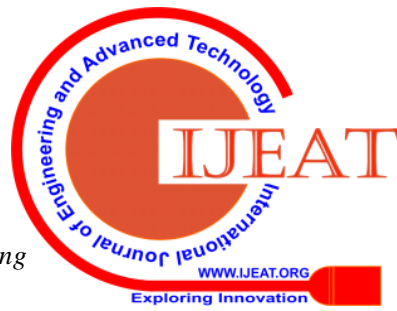

